\title{
DIVERSIDAD DE MURCIÉLAGOS (CHIROPTERA: MAMMALIA) EN DOS ZONAS ARQUEOLÓGICAS DE YUCATÁN, MÉXICO
}

\author{
Erendira ESTRElla, ${ }^{1}$ Juan M. PECH-CANCHÉ, ${ }^{2,3}$ Silvia F. \\ HERNÁNDEZ-BETANCOURT, ${ }^{1}$ Diana L. LÓPEZ-CASTILLO ${ }^{1}$ \& ClaUdia \\ E. MORENO ${ }^{4}$ \\ ${ }^{1}$ Departamento de Zoología, Facultad de Medicina Veterinaria y Zootecnia, Universidad Autónoma de \\ Yucatán, Apartado Postal 4-116, ltzimná, Mérida, Yucatán, México \\ ${ }^{2}$ Red de Ecoetología, Instituto de Ecología, A.C., A.P. 63, Xalapa 91000, Veracruz, México \\ ${ }^{3}$ Dirección actual: Facultad de Ciencias Biológicas y Agropecuarias, Universidad Veracruzana, Km. \\ 7.5 Carretera Tuxpan-Tampico, Colonia Universitaria, Tuxpan, Veracruz, C.P. 92850, México; E-mail: \\ $<$ jmpech@gmail.com> \\ ${ }^{4}$ Centro de Investigaciones Biológicas, Universidad Autónoma del Estado de Hidalgo, Apdo. Postal \\ 69-1, Pachuca, Hidalgo, México
}

Estrella, E., Pech-Canché, J. M., Hernández-Betancourt, S. F., López-Castillo, D. L. \& Moreno, C. E. 2014. Diversidad de murciélagos (Chiroptera: Mammalia) en dos zonas arqueológicas de Yucatán, México. Acta Zoológica Mexicana (n. s.), 30(1): 188-200.

RESUMEN. Las zonas arqueológicas (ZAs) representan un patrimonio cultural por ser un vestigio material de las culturas de nuestros antepasados; sin embargo, se desconoce su potencial para la conservación biológica. Nuestro objetivo fue evaluar la diversidad de murciélagos en las ZAs Dzibilchaltún y Kabah en Yucatán, México, destacando su potencial para la conservación. Aunque la riqueza de especies no fue significativamente diferente entre ambas ZAs, existen diferencias en la diversidad y la composición de los gremios tróficos que pueden estar asociados con diferencias en las condiciones ambientales de cada ZA. Los resultados de nuestro estudio muestran que éstos sitios albergan un alto procentaje de riqueza de especies, tanto a nivel del estado de Yucatán (41\%) como de la península (28\%), demostrando su importancia como reservorios de diversidad y su potencial para complementar las políticas de conservación.

Palabras clave: Zonas arqueológicas mayas, diversidad de murciélagos, México, Dzibilchaltún, Kabah.

Estrella, E., Pech-Canché, J. M., Hernández-Betancourt, S. F., López-Castillo, D. L. \& Moreno, C. E. 2014. Bat diversity (Chiroptera: Mammalia) in two archaeological sites in Yucatan, Mexico. Acta Zoológica Mexicana (n. s.), 30(1): 188-200. 


\begin{abstract}
The archaeological sites represent a cultural heritage because of the pre-Hispanic constructions they protect; however, its potential for biological conservation is unknown. Our aim was to assess the diversity of bats in the Dzibilchaltún and Kabah archaeological sites in Yucatan, Mexico, highlighting the potential of these sites for conservation. Although species richness was not different between sites, there was variation in bat diversity and the composition of trophic guilds, which might be associated with differences in environmental conditions of each site. The results of our study show that these sites harbor a high percentage of bat richness, both with regard to the state of Yucatan (41\%) as well as to the Yucatan Peninsula (28\%), demonstrating its importance as reservoirs to the diversity of bats and its potential to complement conservation policies.
\end{abstract}

Key words: Mayan archaeological sites, diversity of bats, Dzibilchaltún, Kabah, Mexico.

\title{
INTRODUCCIÓN
}

La conservación biológica tradicionalmente se ha buscado mediante el establecimiento de un sistema de áreas que protejan muestras representativas de los distintos ecosistemas (Halffter 1984). En este sentido, México cuenta actualmente con 176 áreas naturales protegidas (ANPs) de carácter federal que abarcan e1 12.92\% del territorio nacional (CONANP 2013), además de diversas ANPs decretadas por otros niveles de gobierno (estados y municipios) e incluso algunas reservas privadas. Sin embargo, éstas áreas por sí solas no han logrado representar adecuadamente los ecosistemas naturales o la biodiversidad para favorecer la conservación efectiva y a largo plazo, ya que muchas de ellas, principalmente las primeras ANP's, fueron establecidas por su belleza escénica o por otros valores no necesariamente prioritarios para la conservación (Bezaury-Creel \& Gutiérrez 2009; Koleff et al. 2009).

Las zonas arqueológicas (ZAs), como estrategia para la preservación de los espacios naturales, han sido poco exploradas respecto a su valor para el mantenimiento y conservación de la biodiversidad. Las ZAs son sitios de interés antrópico debido a los materiales, muebles y/o inmuebles arqueológicos que albergan, frutos de la acción humana, y que con el paso del tiempo se vuelven parte del entorno natural. Por su relevancia histórica y cultural, muchas de ellas han sido decretadas como zonas sujetas a conservación para la investigación arqueológica, acción que también les permite ser suceptibles para el mantenimiento y conservación de muchas especies de plantas y animales, en especial en zonas donde existieron grandes asentamientos humanos prehispánicos.

En México se cuenta con un total de 187 ZAs sujetas a investigación, protección, conservación y divulgación. La Península de Yucatán resulta una de las regiones con mayor concentración de éstas, con un total de 52 ZAs (20 en Quintana Roo y 16 en Yucatán y Campeche) (INAH 2013). Sin embargo, a pesar de este alto número de sitios sujetos a protección, poco se sabe acerca de la diversidad biológica que mantienen debido a que los objetivos de investigación han sido arqueológicos y no ecológicos. Los estudios faunísticos en las ZAs se han basado principalmente en la descripción de los restos óseos recuperados en estas zonas (Álvarez 1976; Wing \& 
Steadman 1980; Arroyo-Cabrales \& Álvarez 1990) o los usos históricos de la fauna en las mismas (Götz 2008; Masson \& Peraza 2008). Y aunque ya se han realizado algunos estudios de ecología de poblaciones en estos sitios (Scott \& Martin 1983; Ortega \& Martínez-Rodríguez 2011), los trabajos a nivel de comunidades aún son escasos (White \& Darwin 1995), particularmente para murciélagos. En Mesoamérica se presenta un patrón similar, ya que la información sobre su presencia en ZAs se ha basado en registros históricos ocasionales (Kuns \& Tashian 1954; Arroyo-Cabrales \& Álvarez 1990) más que en estudios biológicos sistematizados (Meachem 1968; McCarthy 1982). Recientemente se ha empezado a reconocer la importancia que pudieran tener las ZAs para la conservación de los murciélagos (Avila-Torresagatón et al. 2012), la cual pudiera estar más asociada a la configuración del paisaje en el sitio que a los vestigios arqueológicos per se.

Los murciélagos son un grupo ecológicamente importante debido a la diversidad de procesos ecológicos en los que intervienen, como polinización de plantas, dispersión de semillas y control de poblaciones de insectos (Jones et al. 2009); y debido a que pueden responder a cambios en los ecosistemas producidos por el hombre (Fenton et al. 1992; Medellín et al. 2000) han sido considerados como indicadores ecológicos de calidad de hábitat.

Por lo anterior, el objetivo del presente trabajo fue analizar la diversidad de murciélagos en dos ZAs del estado de Yucatán, México: el Parque Nacional Dzibilchaltún y el Parque Estatal de Kabah. De manera particular, se evaluó la representatividad de los inventarios en cada ZA estimando el número máximo de especies presentes en ellas, se comparó la riqueza, la diversidad y las proporciones de gremios tróficos entre las dos ZAs, y se midió su porcentaje de disimilitud (complementariedad) en la composición de especies. Con base en estos análisis, se discute el potencial que éstas ZAs pueden tener en el mantenimiento de la diversidad de murciélagos, y su relación con la biodiversidad en general.

\section{MATERIALES Y MÉTODOS}

Área de estudio. El trabajo fue realizado en dos ZAs del estado de Yucatán, México: el Parque Nacional Dzibilchaltún y el Parque Estatal de Kabah, las cuales fueron seleccionadas por encontrarse en los dos hábitats naturales más representativos de Yucatán, la selva baja caducifolia y la selva mediana subcaducifolia, respectivamente, además de que se trata de ZAs que cuentan con decreto oficial para su protección (SECOL 1993; 2007), siendo el turismo a pequeña escala el único uso de suelo permitido.

El Parque Nacional Dzibilchaltún $\left(21^{\circ} 05^{\prime} \mathrm{N}-89^{\circ} 36^{\prime} \mathrm{W}\right)$ pertenece al municipio de Mérida, cuenta con un área total de 539 ha y en su interior se encuentra un cuerpo de agua (cenote Xlacah), que abarca $60 \mathrm{~m}^{2}$ de superficie; el clima es cálido subhúmedo con lluvias en verano y sequías a la mitad del año (Flores \& Espejel 1994), 
con una precipitación anual entre 700 y $900 \mathrm{~mm}$ y temperatura media anual de 28 ${ }^{\circ} \mathrm{C}$ (SECOL 1993); el tipo de vegetación dominante es selva baja caducifolia, que cubre el $50 \%$ de la superficie de la ZA, con una altura promedio de $8 \mathrm{~m}$ y una altura máxima del dosel de $13 \mathrm{~m}$, las especies más comunes son: Acacia gaumeri, Mimosa bahamensis, Gymnopodium floribundum, Bursera simaruba y Caesalpinia gaumeri (Flores \& Espejel 1994). El Parque Estatal de Kabah (20¹4' N - 89³9’ W) pertenece al municipio de Santa Elena, cuenta con una superficie de 949 ha (SECOL 2007); el clima es cálido subhúmedo con lluvias en verano (Flores \& Espejel 1994), con una precipitación media anual de 1,062 $\mathrm{mm}$ y una temperatura media anual de 24.8 ${ }^{\circ} \mathrm{C}$; el tipo de vegetación dominante es selva mediana subcaducifolia, que cubre el $35 \%$ de la superficie de la ZA, con una altura promedio de $12 \mathrm{~m}$ y una altura máxima del dosel de $20 \mathrm{~m}$, las especies más comunes son: Caesalpinia gaumeri, Lysiloma latisiliquum, Piscidia piscipula, Acacia cornigera, Bursera simaruba (SECOL 2007).

Métodos de captura. Los muestreos se realizaron durante dos noches consecutivas mensualmente en cada ZA entre Noviembre 2005 y Octubre 2006, evitando las noches de lluvia o luna llena (Morrison 1978). Para la captura de murciélagos se colocaron dos redes de niebla a nivel de suelo (sotobosque), dos redes a una altura de $8 \mathrm{~m}$ (sub-dosel), además de dos trampas arpa en el interior de la vegetación; todas las redes fueron de $12 \mathrm{~m}$ de largo por 2.5 de ancho, con una abertura de malla de $38 \mathrm{~mm}$, en tanto que las trampas arpa fueron de $1.2 \mathrm{~m}$ de alto y $1.0 \mathrm{~m}$ de ancho, con cuatro líneas. Las redes y las trampas permanecieron abiertas durante seis horas a partir del atardecer, y se revisaron cada $30 \mathrm{~min}$. Los individuos capturados fueron identificados mediante guías especializadas (Medellín et al. 1997; Reid 1997) y liberados en el mismo sitio de captura en menos de una hora. El manejo de todos los individuos capturados se realizó de acuerdo a los parámetros éticos establecidos por la American Society of Mammalogists (Sikes et al. 2011).

Análisis de datos. Para evaluar la representatividad de los inventarios se calculó el porcentaje que representa la riqueza de especies observada en cada ZA con relación a la riqueza de especies máxima esperada, calculada esta última como el promedio de los índices ICE, Chao 2 y Boostrap, que son los estimadores menos sesgados con tamaños de muestra pequeños (Colwell et al. 2004); para reconocer como satisfactorio el inventario, se consideró una representatividad de 90\% (Moreno \& Halffter 2000). Para comparar la riqueza acumulada de especies se ajustaron curvas de rarefacción (función de Mao Tau) con intervalos de confianza al 95\%, aleatorizando 1000 veces las muestras para evitar la influencia del orden en que los individuos fueron registrados. La comparación de la riqueza se realizó estandarizando a un mismo tamaño de muestra (el número de individuos de la ZA con menor abundancia). Tanto los estimadores de riqueza como las curvas de rarefacción fueron calculados con el programa EstimateS, version 8.2 (Colwell 2006). 
Para obtener la diversidad alfa en cada ZA se empleó el exponencial del índice de Shannon o diversidad verdadera de orden 1 (Jost 2006), cuyas unidades son el número de especies efectivas en las comunidades (Moreno et al. 2011; García-Morales et al. 2011). Para probar diferencias en las proporciones de gremios tróficos en ambas ZAs, se realizó una prueba de G para tablas de contingencia, subdividiendo dicha tabla por gremios para detectar a los que de manera específica estén asociados a una ZA en particular (Zar 2010); dichos análisis estuvieron basados en el sistema de clasificación propuesto por Simmons y Voss (1998).

Finalmente, para medir la disimilitud en la composición de especies entre ambas ZAs se calculó el índice de complementariedad de Colwell y Coddington (1994), el cual se expresa en porcentaje y varía desde cero cuando ambos sitios son idénticos hasta 100 cuando ambos sitios son completamente distintos.

\section{RESULTADOS}

En total se capturaron 566 individuos pertenecientes a 18 especies de cuatro familias, la familia Phyllostomidae fue la mejor representada (Cuadro 1). Para ambas ZAs el esfuerzo de muestreo puede ser considerado adecuado ya que se registraron valores de representatividad cercanos al 90\% de acuerdo a los estimadores empleados (Cuadro 1). Con relación a la abundancia, el número de individuos capturado en Kabah representa más del $60 \%$ de la captura total de murciélagos en el estudio. Al comparar las ZAs a un tamaño de muestra estandarizado (207 individuos), no se encontraron diferencias significativas en la riqueza total de especies entre ambos sitios de acuerdo con los intervalos de confianza del 95\% (Fig. 1).

La diversidad de especies fue mayor en Dzibilchaltún que en Kabah: 8.09 y 6.7 especies efectivas, respectivamente, lo que significa que esta última alberga el $82.7 \%$ de la diversidad de murciélagos de Dzibilchaltún (Cuadro 1). Las capturas totales de ambos sitios fueron dominadas por cuatro especies: Artibeus jamaicensis (38.7\%), Glossophaga soricina (18\%), A. lituratus y Sturnira parvidens (ambas con 7.8\%), que en conjunto representan $72.3 \%$ de la abundancia total en ambos sitios; seis especies se presentaron en abundancia media (de 24 a 10 individuos), seis en baja (de nueve a cuatro individuos) y dos se consideraron raras (un individuo); de estas especies raras, una fue registrada en Dzilbilchaltún (Natalus stramineus) y otra en Kabah (Mimon cozumelae).

La proporción de individuos de cada gremio fue significativamente diferente entre ambos sitios $\left(G^{2}=31.08, \mathrm{p}<0.001\right)$; aunque los frugívoros fueron el gremio mejor representado en todo el estudio (7 especies y 392 individuos), sólo el gremio de los insectívoros fue proporcionalmente mayor en Dzibilchaltún que en Kabah $\left(G^{2}=\right.$ 23.51, p $<0.001$; Fig. 2).

De las 18 especies totales, 13 (72\%) fueron compartidas entre ambas zonas arqueológicas, por lo que el porcentaje de complementariedad entre ambas zonas fue de 
Cuadro 1. Diversidad de murciélagos presentes en las zonas arqueológicas de Dzibilchaltún y Kabah, Yucatán, México. ${ }^{*} \mathrm{~S}=$ redes de sotobosque; $\mathrm{D}=$ redes de sub-dosel; $\mathrm{A}=$ trampas arpa.

\begin{tabular}{|c|c|c|c|c|}
\hline Taxon & Dzibilchaltún & Kabah & $\underset{*}{\text { Método de registro }}$ & Gremio trófico \\
\hline \multicolumn{5}{|l|}{ PHYLLOSTOMIDAE } \\
\hline Desmodus rotundus & 7 & 3 & $\mathrm{~S}$ & Hematófago \\
\hline Diphylla ecaudata & 6 & 2 & S, D & Hematófago \\
\hline Glossophaga soricina & 32 & 70 & $\mathrm{~S}, \mathrm{D}, \mathrm{A}$ & Nectarívoro \\
\hline Micronycteris microtis & 1 & 5 & S, D & Carnívoro \\
\hline Mimon cozumelae & & 1 & $\mathrm{~S}$ & Carnívoro \\
\hline Carollia sowelli & 10 & 12 & S, D & Frugívoro \\
\hline Carollia perspicillata & & 24 & $\mathrm{~S}, \mathrm{~A}$ & Frugívoro \\
\hline Sturnira parvidens & 16 & 38 & S, D & Frugívoro \\
\hline Artibeus jamaicensis & 80 & 139 & S, D & Frugívoro \\
\hline Artibeus lituratus & 7 & 37 & S, D & Frugívoro \\
\hline Dermanura phaeotis & 2 & 17 & S, D & Frugívoro \\
\hline Centurio senex & 2 & 18 & $\mathrm{~S}, \mathrm{D}, \mathrm{A}$ & Frugívoro \\
\hline \multicolumn{5}{|l|}{ MORMOOPIDAE } \\
\hline Mormoops megalophylla & 7 & & A & Insectívoro \\
\hline Pteronotus parnellii & 8 & 1 & $\mathrm{~S}, \mathrm{~A}$ & Insectívoro \\
\hline Pteronotus davyi & 20 & & A & Insectívoro \\
\hline \multicolumn{5}{|l|}{ NATALIDAE } \\
\hline Natalus stramineus & 1 & & A & Insectívoro \\
\hline \multicolumn{5}{|l|}{ VESPERTILIONIDAE } \\
\hline Rhogeessa tumida & 3 & 1 & A & Insectívoro \\
\hline Myotis elegans & 5 & 1 & $\mathrm{~S}, \mathrm{D}, \mathrm{A}$ & Insectívoro \\
\hline Abundancia & 207 & 359 & & \\
\hline Riqueza de especies & 16 & 15 & & \\
\hline Diversidad $\left[\exp \left(\mathrm{H}^{\prime}\right)\right]$ & 8.08 & 6.69 & & \\
\hline Representatividad (\%) & 92 & 86 & & \\
\hline
\end{tabular}

$28 \%$; de manera exclusiva, en Dzibilchaltún se registraron tres especies insectívoras (Mormoops megalophylla, Pteronotus davyi y Natalus stramineus), mientras que en Kabah sólo se registraron dos especies de filostómidos no compartidas con Dzibilchaltún (Carollia perspicillata y Mimon cozumelae) (Cuadro 1). 


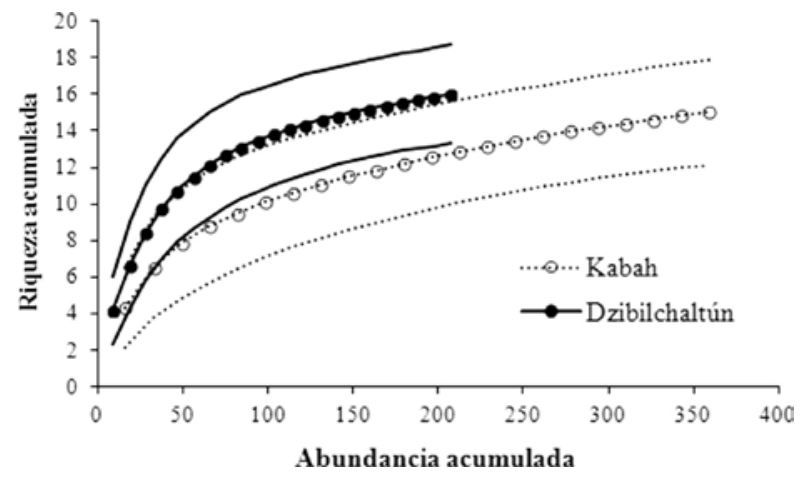

Figura 1. Curvas de rarefacción de especies de murciélagos y sus intervalos de 95\% de confianza en las zonas arqueológicas Dzibilchaltún y Kabah en Yucatán, México.

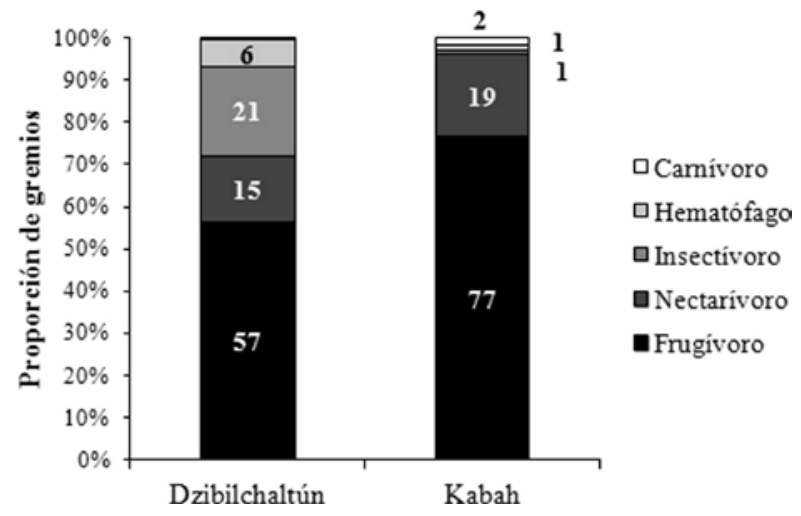

Figura 2. Proporción de murciélagos de cada gremio trófico registrado en las zonas arqueológicas Dzibilchaltún y Kabah en Yucatán, México.

\section{DISCUSIÓN}

\section{Diversidad de murciélagos en las zonas arqueológicas de Dzibilchaltún y Kabah}

El presente estudio es el primero en analizar de manera sistemática la diversidad de murciélagos en dos ZAs de la región norte de la Península de Yucatán, ya que los estudios previos con murciélagos en ZA's han sido basados en registros ocasionales (Sánchez et al. 1986) o análisis de ecología de poblaciones (Ortega \& Martínez-Rodríguez 2011). La riqueza de especies de murciélagos registrada de forma acumulada en ambas zonas arqueológicas de Dzibilchaltún y Kabah (18 especies) representa 
prácticamente la mitad de la riqueza de especies de murciélagos reportada para el estado de Yucatán (44 especies) y el 28\% de la riqueza de especies murciélagos que se distribuyen en la península de Yucatán (64 especies; Sosa-Escalante et al. 2013), lo que evidencia la importancia que estas ZAs, y su vegetación asociada, tienen en el mantenimiento de la diversidad de murciélagos a nivel local. A nivel regional, la riqueza de especies de murciélagos registrada en los sitios analizados puede considerarse como media, ya que presenta mayor riqueza de especies que unas ANPs, como Cuxtal, Yucatán (12 especies; Cervantes 2001), similar a otras como El Edén, Quintana Roo (15 especies; MacSwiney 2000), Los Petenes, Campeche (16 especies; Montiel et al. 2007) y Dzilam, Yucatán (20 especies; Segovia 1995), pero menor que otras como Sian Ka'an, Quintana Roo (34 especies; Pozo de la Tijera \& Escobedo 1999) y Calakmul, Campeche (46 especies; Arroyo-Cabrales et al. 2011).

Sin embargo, a pesar de que se alcanzó un adecuado porcentaje de representatividad (cercano al 90\%), la riqueza de especies registrada en ambas ZAs podría estar subestimada debido al uso exclusivo de técnicas de captura y la carencia de un método de registro indirecto (detector ultrasónico), técnica con la cual se podría incrementar el registro de especies entre 30 y 40\% (MacSwiney et al. 2009; Pech-Canche et al. 2010). Registros previos en estas ZAs (Bowles et al. 1990; Arita 1996; Lim et al. 2004; Pech-Canche et al. 2010) reportan especies adicionales a las registradas en este trabajo, por lo cual la diversidad histórica de murciélagos en conjunto en ambas ZAs podría estimarse hasta en 30 especies, que potencialmente se podrían registrar utilizando métodos de muestreo complementarios (27 en Dzibilchaltún y 17 en Kabah).

En nuestro trabajo con redes de niebla la riqueza total de especies fue de solo una especie más en Dzibilchaltún que en Kabah, sin embargo, al unir información de la riqueza y la abundancia relativa de las especies se encontraron diferencias significativas entre ambas ZAs, siendo la diversidad de Dzibilchaltún 1.2 veces mayor que la de Kabah, debido a que en Kabah se presentó una baja equitatividad en la distribución de abundancias y un mayor número de especies raras (especies con sólo un individuo). La mayor proporción de murciélagos insectívoros en Dzibilchaltún puede deberse a que este sitio alberga un cuerpo de agua que puede ser usado como sitio de forrajeo por las especies insectívoras (MacSwiney et al. 2009), además de que la vegetación asociada a los cuerpos de agua provee de sitios de alimentación y perchas temporales nocturnas que pueden ser usadas por éstas u otras especies con diferentes hábitos alimenticios (MacSwiney et al. 2007). La riqueza de plantas leñosas y desarrollo vertical en Kabah (Ortiz-Díaz, datos no publicados), aportan alimento y refugio, así como un hábitat mas estructurado que permite la coexistencia de una mayor diversidad de especies (Bernard 2001), en su mayoría fitófagas (frugívoras y nectarívoras). Un ejemplo es $C$. perspicillata, una especie con tamaños poblacionales pequeños en la región (MacSwiney et al. 2007) y asociada a cuevas con alta riqueza de especies (Arita \& Vargas 1995), cuyo registro exclusivo en Kabah puede deberse no sólo a las 
características de la vegetación sino a su ubicación en la Región Puuc (Arita \& Vargas 1995), zona con una gran cantidad de grutas que pueden ser usadas como sitios de refugio por muchas especies de murciélagos (Arita 1996).

El bajo porcentaje de complementariedad entre las dos ZAs estudiadas indica que la composición de especies de murciélagos es similar, lo cual es consistente con la baja tasa de recambio de especies en la Península de Yucatán con relación a otras regiones de México (Arita \& Rodríguez 2002); sin embargo, ciertas diferencias se aprecian a nivel taxonómico, ya que en Kabah se registraron más especies de la familia Phyllostomidae mientras que en Dzibilchaltún se tuvo un mayor registro de especies insectívoras de otras familias, lo que demuestra la importancia del uso combinado de diferentes técnicas de registro de especies (Pech-Canche et al. 2010).

\section{Importancia de las zonas arqueológicas para la conservación}

Las ZAs pueden ser elementos muy importantes para complementar las políticas de conservación basadas principalmente en el establecimiento de ANPs, ya que, debido a la vegetación asociada que mantienen, pueden llegar a albergar una gran riqueza de especies, como en este trabajo se documenta para el caso de murciélagos en el estado de Yucatán, y otros estudios lo han demostrado en otras regiones de México (AvilaTorresagatón et al. 2012).

Un ejemplo a nivel regional es la ZA de Calakmul, en Campeche, que puede considerarse como uno de los primeros esfuerzos para promover la conservación de la diversidad a través de la protección de las ZAs, ya que se estableció para conservar uno de los agrupamientos más sobresalientes de la cultura Maya, pero su gran extensión (723,185 ha) y la diversidad de hábitats que alberga (INE 2000), ha permitido que en esta zona se conserven 46 especies de murciélagos (Arroyo-Cabrales et al. 2011), que representan el $72 \%$ de la riqueza de murciélagos presente en la península de Yucatán. Otra ZA de gran importancia histórica en la región es Chichén Itzá, en la cual las colectas de murciélagos datan desde finales del siglo XVIII y el registro histórico actual de murciélagos es de 20 especies (Pech-Canché, datos no publicados), reconociéndola como un punto importante en el mantenimiento de la diversidad de murciélagos al albergar aproximadamente una tercera parte de los murciélagos de la península de Yucatán, a pesar de su pequeña extensión (1,547 ha) y la falta de estudios formales que permitan una adecuada caracterización de la diversidad de murciélagos que alberga.

Considerando que la función principal de las ZAs es la protección de los vestigios arqueológicos, una de las medidas para minimizar el daño a los monumentos históricos por actividades antrópicas es la regulación del turismo y de los espectáculos de luz y sonido. Estos factores pueden afectar físicamente los vestigios arqueológicos (Garduño 2009), además de modificar la biología de las especies de murciélagos mediante la alteración de sus patrones de actividad nocturna (Shirley et al. 2001), ya 
que el retrasar su emergencia puede ocasionar un desfase con la disponibilidad de los insectos de los cuales se alimentan, lo cual tiene implicaciones directas en su sobrevivencia. Aunque la acumulación de guano de murciélagos puede ocasionar daños a los vestigios arqueológicos, esto sucede principalmente en grupos arquitectónicos grandes, poco visitados y con estructuras cerradas como bóvedas o túneles (Martínez 2001), por lo que las características de las ZAs analizadas (sitios pequeños, abiertos y con flujo de aire continuo) no favorecen su uso como sitios de percha.

Además de la riqueza de especies que las ZAs pueden mantener, su importancia ecológica podría ser mayor si se consideran como corredores biológicos que permitan la conectividad entre las grandes ANPs que se encuentran en los extremos de la península de Yucatán, ya que existe evidencia de que las ZAs de la Región Puuc pueden permitir la conexión genética entre poblaciones de murciélagos distantes (Morales 2009).

La conservación y el mantenimiento de la riqueza arqueológica y la diversidad biológica, son procesos que deberían hacerse compatibles a fin de que se complementen y se maximicen los resultados que podrían obtenerse de manera aislada, por lo cual, el desarrollo de trabajos de este tipo permite demostrar que la dualidad entre la conservación de la diversidad biológica y cultural pueden desarrollarse con una visión integradora que mantenga a largo plazo la viabilidad de la biodiversidad y la diversidad sociocultural en un mismo espacio natural.

AGRAdeCimientos. A la Universidad Autónoma de Yucatán, que a través del proyecto de Fondos Sectoriales FOSEMARNAT-2004-01-180 financió parcialmente el trabajo. Al Instituto Nacional de Antropología e Historia, Delegación Yucatán, por habernos permitido ingresar a las zonas arqueológicas. A los guardaparques de ambas zonas arqueológicas por su apoyo logístico, así como a todas las personas que nos acompañaron en el trabajo de campo.

\section{LITERATURA CITADA}

Álvarez, T. 1976. Restos óseos rescatados del cenote sagrado de Chichén Itzá, Yucatán. Cuadernos de trabajo del INAH, 15: 19-39.

Arita, H. T. 1996. The conservation of cave-roosting bats in Yucatán, México. Biological Conservation, 76: 177-185.

Arita, H. T. \& Rodríguez, P. 2002. Geographic range, turnover rate and the scaling of species diversity. Ecography, 25: 541-550.

Arita, H. T. \& Vargas, J. 1995. Natural history, interspecific association and incidence of the cave bats of Yucatan, Mexico. The Southwestern Naturalist, 40: 29-37.

Arroyo-Cabrales, J. \& Álvarez, T. 1990. Restos óseos de murciélagos procedentes de las excavaciones en las grutas de Loltún. INAH, Serie Prehistoria, Colección Científica. México.

Arroyo-Cabrales, J., González-Christen, A., Canales, D., León, F., Franco, M. L., Navarro, L. \& Vargas-Contreras, A. 2011. Los murciélagos de Calakmul. UV, CoVeCyT, CFE. México.

Avila-Torresagatón, L. G., Hidalgo-Mihart, M. \& Guerrero, J. A. 2012. La importancia de Palenque Chiapas, para la conservación de los murciélagos de México. Revista Mexicana de Biodiversidad, 83: 184-193. 
Bernard, E. 2001. Vertical stratification of bat communities in primary forests of Central Amazon, Brazil. Journal of Tropical Ecology, 17: 115-126.

Bezaury-Creel, J. \& Gutiérrez, D. 2009. Áreas naturales protegidas y desarrollo social en México, pp. 385-431 In: Dirzo R, González, R. \& March, I. J. (Eds.). Capital natural de México, vol. II: Estado de conservación y tendencias de cambio. CONABIO, México.

Bowles, J., Heideman, P. \& Erickson, K. 1990. Observations on six species of free-tailed bats (Molossidae) from Yucatan, Mexico. Southwestern Naturalist, 35: 151-157.

Cervantes, M. C. 2001. Estructura y diversidad de la comunidad de quirópteros en tres sitios con perturbación antropogénica en la Reserva Ecológica Cuxtal, Yucatán, México. Tesis de Licenciatura. Universidad Autónoma de Yucatán. México.

Colwell, R. K. 2006. EstimateS: Statistical estimation of species richness and shared species from samples. Version 8. < purl.oclc.org/estimates>

Colwell, R. K., Mao, C. X. \& Chang, J. 2004. Interpolating, extrapolating and comparing incidencebased species accumulation curves. Ecology, 85: 2717-2727.

Colwell, R. K. \& Coddington, J. A. 1994. Estimating terrestrial biodiversity through extrapolation. Philosophical Transactions of the Royal Society of London, series Biological Sciences, 345: 101118.

Comisión Nacional de Áreas Naturales Protegidas (CONANP). 2013. Áreas Protegidas Decretadas. (Consultado 2 de agosto de 2013, http://www.conanp.gob.mx/que_hacemos/).

Fenton, M. B., Acharya, L., Audet, D., Hickey, M. B., Merriman, C., Obrist, M. K., Syme, D. M. \& Adkins, B. 1992. Phyllostomyd bats (Chiroptera: Phyllostomidae) as indicators of habitat disruptions in the Neotropics. Biotropica, 24: 440-446.

Flores, J. \& Espejel, I. 1994. Tipos de vegetación de la península de Yucatán. Etnoflora Yucatanense. Universidad Autónoma de Yucatán. Yucatán, México.

García-Morales, R., Moreno, C. E. \& Bello-Gutiérrez, J. 2011. Renovando las medidas para evaluar la diversidad en comunidades ecológicas: el número de especies efectivas de murciélagos en el sureste de Tabasco, México. Therya, 2: 205-215.

Garduño, J. 2009. El show o espectáculo de luz y sonido en patrimonio arqueológico a debate: ¿es positivo? ¿es negativo? III Congreso Internacional de Antropología desde la frontera sur. Quintana Roo, México.

Götz, C. M. 2008. Coastal and inland patterns of faunal exploitation in the prehispanic northern Maya lowlands. Quaternary International, 191: 154-169.

Halffter, G. 1984. Las reservas de la biósfera: conservación de la naturaleza para el hombre. Acta Zoológica Mexicana (n.s.), 5: 4-48.

Instituto Nacional de Antropología e Historia (INAH). 2013. Red de Zonas arqueológicas. (Consultado 2 de agosto de 2013, http://www.inah.gob.mx/index.php/zonas-arqueologicas).

Instituto Nacional de Ecología (INE). 2000. Programa de manejo Reserva de la Biosfera Calakmul. Instituto Nacional de Ecología. México, D.F.

Jones, G., Jacobs, D. S., Kunz, T. H., Willig, M. R. \& Racey, P. A. 2009. Carpe noctem: the importance of bats as bioindicators. Endangered Species Research, 8: 93-115.

Jost, L. 2006. Entropy and diversity. Oikos, 113: 363-375.

Koleff, P., Tambutti, M., March, I., Esquivel, R., Cantú, C. \& Lira-Noriega, A. 2009. Identificación de prioridades y análisis de vacíos y omisiones en la conservación de la biodiversidad de México, pp. 651-718 In: Dirzo, R., González, R. \& March, I. J. (Eds.). Capital natural de México, vol. II: Estado de conservación y tendencias de cambio. CONABIO, México.

Kuns, M. \& Tashian, R. 1954. Notes on mammals from northern Chiapas, Mexico. Journal of Mammalogy, 35: 100-103. 
Lim, B. K., Engstrom, M. D., Simmons, N. B. \& Dunlop, J. M. 2004. Phylogenetics and biogeography of least sac-winged bat (Balantiopteryx) based on morphological and molecular data. Mammalian Biology, 69: 225-237

MacSwiney, M. C. 2000. Estructura de la comunidad de quirópteros de la reserva ecológica de "El Edén”, Quintana Roo, México. Tesis de Licenciatura. Universidad Autónoma de Yucatán. México.

MacSwiney, M. C., Vilchis, P., Clarke, F. M. \& Racey, P. A. 2007. The importance of cenotes in conserving bat assemblages in the Yucatan, Mexico. Biological Conservation, 136: 499-509.

MacSwiney, M. C., Bolivar, B., Clark, F. M. \& Racey, P. A. 2009. Insectivorous bat activity at cenotes in the Yucatan Peninsula, México. Acta Chiropterologica, 11: 139-147.

Martínez, G. A. 2001. La ruina arqueológica como mundo viviente: La convivencia biocultural en el Parque Nacional Tikal, pp. 210-220 In: Laporte, J .P., Suasnávar, A. C. \& Arroyo, B. (Eds.). XIV Simposio de Investigaciones Arqueológicas en Guatemala, 2000. Museo Nacional de Arqueología y Etnología, Guatemala.

Masson, M. A. \& Peraza, C. 2008. Animal use at the Postclassic Maya center of Mayapan. Quarternary International, 191: 170-183.

McCarthy, T. J. 1982. Bat records from the Caribbean Lowlands of El Peten, Guatemala. Journal of Mammalogy, 63: 683-685.

Meachem, A. R. 1968. Notes on bats from Tikal, Guatemala. Journal of Mammalogy, 49: 516-520.

Medellín, R., Arita, H. T. \& Sánchez, O. 1997. Identificación de los murciélagos de México. Clave de campo. AMMAC, A.C. Publicaciones especiales \# 2. México.

Medellín, R., Equihua, M. \& Amín, M. A. 2000. Bat diversity and abundance as indicators of disturbance in Neotropical rainforest. Conservation Biology, 14: 1666-1675.

Morales, A. 2009. Estructura y diversidad genética de Nyctinomops laticaudatus (Chiroptera: Molossidae) en el Estado de Yucatán, México. Tesis de Licenciatura. Universidad Autónoma del Estado de México, México.

Moreno, C. \& Halffter, G. 2000. Assessing the completeness of bat biodiversity inventories using species accumulation curves. Journal of Applied Ecology, 37: 149-158.

Moreno, C. E., Barragán, F., Pineda, E. \& Pavón, N. P. 2011. Reanálisis de la diversidad alfa: alternativas para interpretar y comparar información sobre comunidades ecológicas. Revista Mexicana de Biodiversidad, 82: 1249-1261.

Morrison, D. W. 1978. Lunar phobia in a Neotropical fruit bat, Artibeus jamaicensis (Chiroptera: Phyllostomidae). Animal Behavior, 26: 852-855.

Montiel, S., León, P. \& Estrada, A. 2007. Riqueza y diversidad de quirópteros en hábitats-isla en una región naturalmente fragmentada de Mesoamérica, pp. 373-392. In: Harvey, C. A. \& Sáenz, J. C. (Eds.). Evaluación y conservación de biodiversidad en paisajes fragmentados de Mesoamérica. Editorial INBio. Costa Rica.

Ortega, J. \& Martínez-Rodríguez, J. L. 2011. Conductas de apareamiento y agresión entre machos en una colonia de Nyctinomops laticaudatus (Chiroptera: Molossidae) en México. Mastozoología Neotropical, 18: 95-103.

Pech-Canché, J. M., MacSwiney, M. C. \& Estrella, E. 2010. Importancia de los detectores ultrasónicos para mejorar los inventarios de murciélagos Neotropicales. Therya, 1: 221-228.

Pozo de la Tijera, C. \& Escobedo, J. 1999. Mamíferos terrestres de la Reserva de la Biosfera de Sian Ka'an, Quintana Roo, México. Revista de Biología Tropical, 47: 251-262

Reid, F. A. 1997. A field guide to the mammals of Central America and southeast Mexico. Oxford University Press. USA.

Sánchez, H., Tellez, G., Medellín, R. \& Urbano, G. 1986. New records of mammals from Quintana Roo, México. Mammalia, 50: 275-278. 
Scott, P. E. \& Martin, R. F. 1983. Reproduction of the turquoise-browed motmot at archaeological ruins in Yucatan. Biotropica, 15: 8-14.

Secretaría de Ecología (SECOL). 1993. Plan de Manejo del Parque Nacional Dzibilchaltún. Gobierno del Estado de Yucatán, Biocenosis, A.C. México.

Secretaría de Ecología (SECOL). 2007. Programa de Manejo, Parque Estatal Kabah. Gobierno del Estado de Yucatán, Universidad Autónoma de Yucatán. México.

Segovia, A. 1995. Los murciélagos de la Reserva de Dzilam, Yucatán, México. Tesis de Licenciatura. Universidad Autónoma de Yucatán. México.

Shirley, M. D. F., Armitage, V. L., Barden, T. L., Gough, M., Lurz, P. W. W., Oatway, D. E., South, A. B. \& Rushton, S. P. 2001. Assessing the impact of a music festival on the emergent behaviour of a breeding colony of Daubenton's bats (Myotis daubentonii). Journal of Zoology, 245: 367-373.

Sikes, R. S., Gannon, W. L. \& The Animal Care and Use Committee of the American Society of Mammalogists. 2011. Guidelines of the American Society of Mammalogists for the use of wild mammals in research. Journal of Mammalogy, 92: 235-253.

Simmons, N. B. \& Voss, R. S. 1998. The mammals of Paracou, French Guiana: A Neotropical lowland rainforest fauna. Part 1. Bats. Bulletin of the American Museum of Natural History, New York, US. No. 237.

Sosa-Escalante, J. E., Pech-Canché, J. M., MacSwiney, M. C. \& Hernández-Betancourt, S. F. 2013. Mamíferos Terrestres de la Península de Yucatán, México: Riqueza, Endemismo y Riesgo. Revista Mexicana de Biodiversidad, 84, DOI: 10.7550/rmb.33285.

White, D. A. \& Darwin, S. P. 1995. Woody vegetation of tropical lowland deciduous forests and Mayan Ruins in the north-central Yucatan Peninsula, Mexico. Tulane Studies in Zoology and Botany, 30: $1-25$.

Wing, E. S. \& Steadman, D. W. 1980. Vertebrate faunal remains from Dzibilchaltún, pp. 326-331 In: Andrews, IV, E. W. \& Andrews V., E. W. (Eds.). Excavations at Dzibilchaltún, Yucatan, Mexico. Middle American Research Institute Publication 48, Tulane University, New Orleans, LA.

Zar, J. 2010. Biostatistical analysis. Prentice Hall. 5th. USA. 